\title{
Encephalocraniocutaneous Lipomatosis: A Case With Long Term Follow-up
}

\author{
Hali Bauld, P. Daniel McNeely
}

Can J Neurol Sci. 2014; 41: 106-108

Encephalocraniocutaneous lipomatosis (ECCL) is a rare neurocutaneous syndrome identifiable by the presence of psiloliparus naevi, ocular abnormalities such as choristomas and colobomas, and central nervous system abnormalities. ${ }^{1}$ Since the first case was reported in 1970 by Haberland and Peru, there have been 60 documented cases worldwide. ${ }^{2}$ The genetic origins of the disease appear to be mosaic and not traceable to one gene or mutation. The disorder is one of mesenchymal origin affecting mostly neural crest cell derivatives. ${ }^{2}$

The hallmark lesion of ECCL is the central nervous system (CNS) lipoma. ${ }^{2}$ Central nervous system lipomas were identified in 33 of the 54 cases reviewed by Moog in 2009. Intracranial lipomas were found frequently at the cerebello-pontine angle and spinal lipomas were identified in 12 of 14 patients who had spinal imaging. Arachnoid cysts were another common CNS finding along with asymmetric changes of the hemispheres and ventricles. ${ }^{1}$ There were also abnormalities noted in the cerebral vessels, including lepto-meningeal angiomatosis, detected only when vascular studies had been performed. ${ }^{1}$

Although there is much known about the clinical features of this syndrome, there is less known about the long term prognosis associated with this diagnosis. We report a case with initial presentation and 12 years of follow-up.

\section{Case Report}

Past Medical History and Physical Exam findings: This 12year-old boy was first seen at our centre as a neonate and has been followed since that time. There was seizure activity noted in the immediate neonatal period that brought him to the attention of the neonatologist. His physical exam findings included a superficial lipoma under the skin involving the sacralanal area measuring eight by three centimeters and a left epibulbar dermoid with a notched eyelid. A review by ophthalmology documented left optic nerve hypoplasia, atrophic retina, left coloboma, and a left corneal dermoid. Dermatological examination demonstrated an oval patch in the left frontoparietal area of his scalp with alopecia and atrophy. He was noted to have urogential abnormalities including an extra renal pelvis and absent left testicle.

On neurological exam, he was found to be hyperreflexic in the lower limbs with sustained clonus in the ankles. He was noted to have a paucity of arm movements and was lacking a Moro reflex. His mother reported that he crawled at two yearsof-age and then had a seated walker until he was four years old. He currently ambulates using a power wheelchair. He has significant contractures and spasticity. The patient has had normal cognitive development and was thought to have been in remission of his epilepsy until the summer of 2011 when he

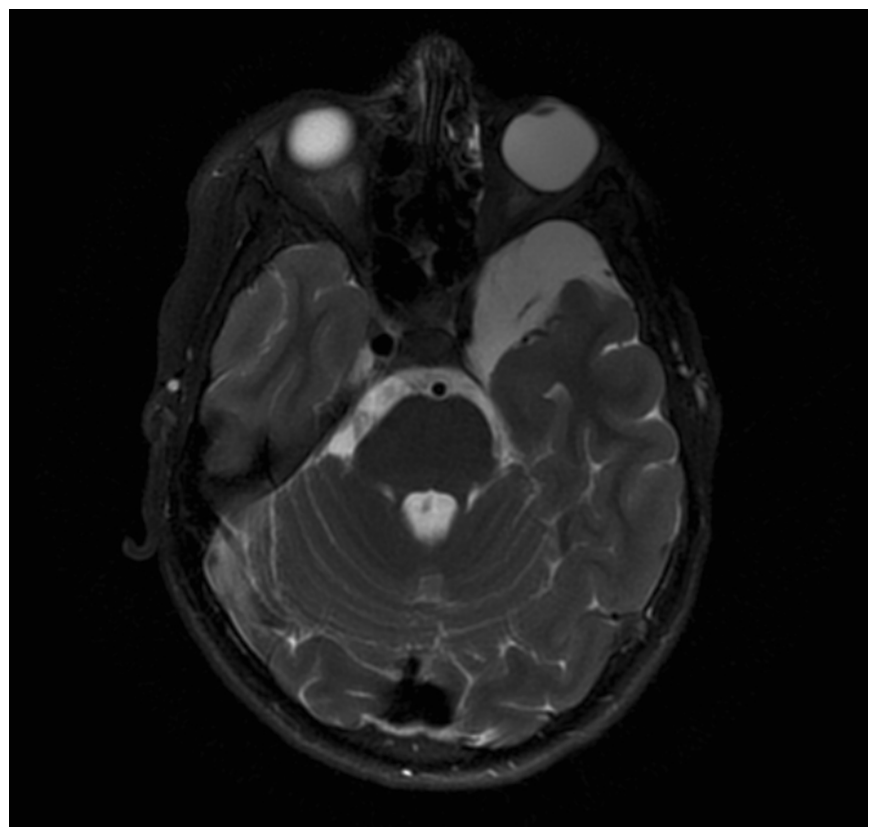

Figure 1: MRI axial T2 weighted image demonstrating a left sylvian fissure arachnoid cyst.

experienced one generalized tonic-clonic seizure and described what may have been simple partial seizures in the preceding months. In terms of other evolving symptomology, the patient has a severe cervical thoracic kyphoscoliosis.

Diagnostic Imaging: He has had diagnostic imaging throughout his course that, on magnetic resonance imaging, has demonstrated a stable left middle cranial fossa arachnoid cyst (Figure 1), platybasia without basilar invagination and an extensive intraspinal lipoma. (Figure 2) The initial image was obtained only four days after the child's birth revealed a lipoma. The rostral extent of the lipoma extended $5 \mathrm{~mm}$ into the posterior fossa and extended caudally to $\mathrm{L} 2 / 3$ disc space. The next

From the Division of Neurosurgery, Department of Surgery, IWK Health Centre, Dalhousie University, Halifax, Nova Scotia, Canada.

Received June 6, 2013. Final Revisions Submitted July 19, 2013.

Correspondence to: P. Daniel McNeely, Division of Neurosurgery, IWK Health Centre, 5850 University Ave., P.O. Box 9700, Halifax, Nova Scotia, B3K 6R8, Canada. Email: dmcneely@dal.ca. 


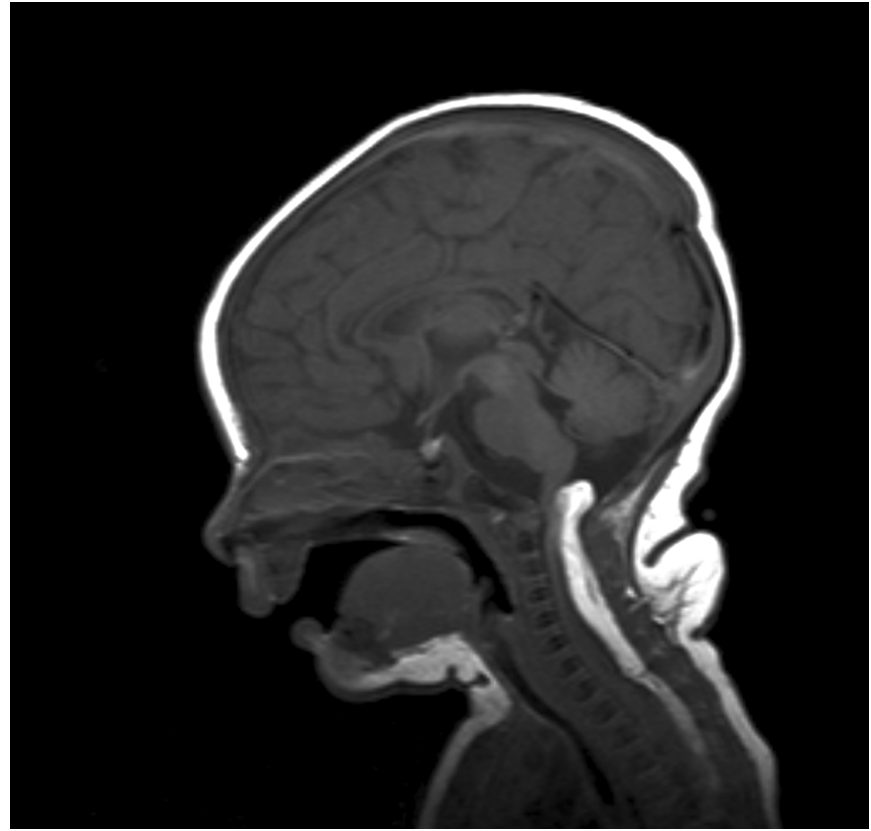

Figure 2: MRI Sagittal T1 weighted image on day 4 of life demonstrating the intraspinal lipoma that projects $5 \mathrm{~mm}$ into the posterior fossa.

imaging studies obtained in 2001 demonstrated significant progression of the lipoma now extending rostrally $20 \mathrm{~mm}$ into the posterior fossa down to the L3 vertebral level. (Figure 3) On imaging studies from birth, the patient's spinal alignment was normal. Imaging performed during childhood demonstrated a developing kyphoscoliosis that continues to worsen on imaging studies performed in 2011. (Figure 4)

Surgeries: Surgical intervention was pursued to deal with several of his physical abnormalities and includes a surgery on his left eye, a nephrectomy due to persistent urinary tract infections, Achilles tendon release and surgery to correct scoliosis in 2008. The patient's scoliosis remains a problem and there is ongoing discussion as to the course of management for this progressive process. (Figure 5) Surgery was never performed for his extensive intraspinal lipoma.

\section{Discussion}

Encephalocraniocutaneous lipomatosis is a disorder that has several hallmarks features that present consistently across the 60 cases that have been reported in the literature to date. Other features such as scoliosis, quadriparesis and their accompanying clinical course are less well documented.

Our patient presented with several of the features identified by Hunter meeting the criteria for a diagnosis of ECCL. ${ }^{3}$ There are reports of mild spastic hemiparesis in these patients, however quadriparesis, as seen in our patient does not appear to be a common feature in this disease. ${ }^{4}$ The mechanism of this patient's quadriparesis is unclear. His neurological deficits have been stable over time. Dysgenesis of the spinal cord due to the presence of the lipoma may be a factor. Spinal cord compression from the extensive lipoma may have played a causative role as well.

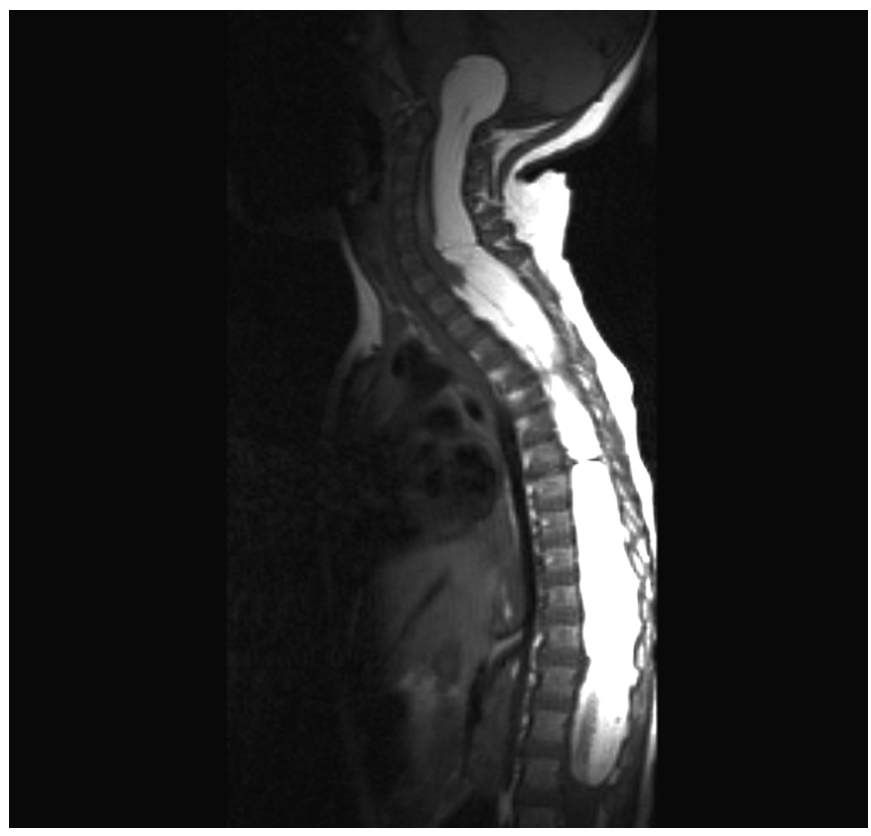

Figure 3: MRI depicting A: Sagittal T1 weighted image obtained in 2001 of spinal lipoma extending $20 \mathrm{~mm}$ into the posterior fossa and normal vertebral alignment.

Deciding on surgical management with respect to the spinal cord lipoma is challenging in the face of paucity of evidence due to the relatively rarity of this finding. In our patient it was decided that conservative management was the best way to proceed. In the review of the literature detailing intradural spinal

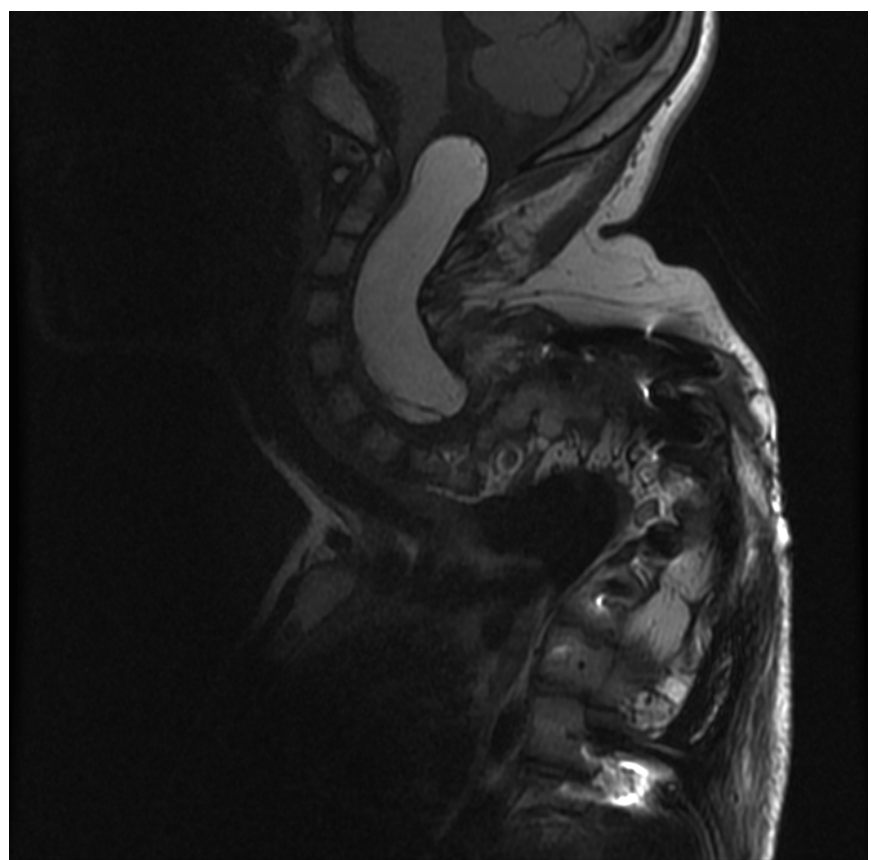

Figure 4: Image taken in August 2011 showing regression of the rostral part of lipoma extending $6 \mathrm{~mm}$ into the posterior fossa. Progression of the kyphoscoliosis is also seen. 


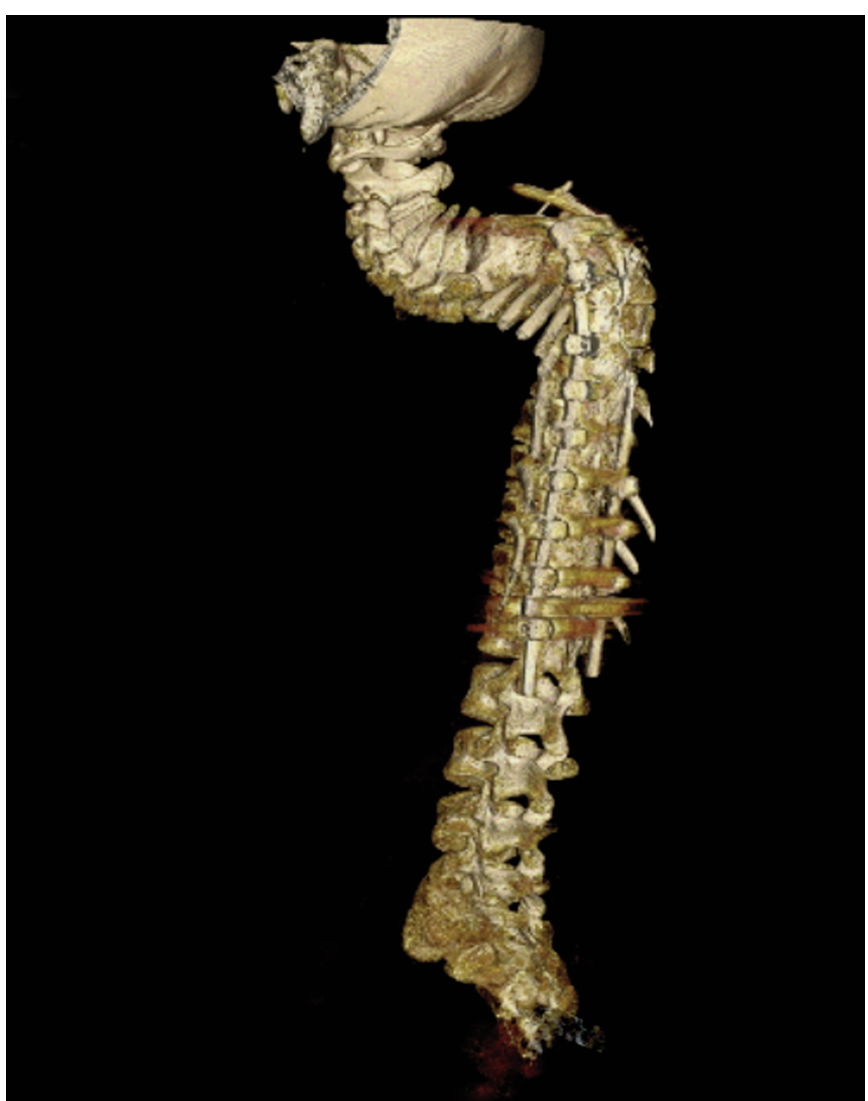

Figure 5: Three-dimensional reconstruction of a computed tomography study obtained at 12 years-of-age demonstrating a severe cervicothoracic scoliosis.

lipomas with intracranial extension where surgical decompression was undertaken, 5 out of 13 patients showed functional improvement, 6 showed no functional improvement, 1 remained at baseline and 1 died..$^{5}$ Given this data, there is no clear answer as to whether or not patients will benefit from surgical intervention. In the case reported by Ayer and Zourous of a child with ECCL and spinal lipoma, surgery was undertaken and some functional improvement of quadriparetic symptoms however post operative complications necessitated the placement of a cerebrospinal fluid shunt and the patient's scoliosis continued to limit the patient's ability to walk. ${ }^{2}$

With respect to our patient's severe scoliosis, there has only been one previously reported case of ECCL with accompanying scoliosis and this is the case noted above by Ayer and Zourous. ${ }^{2}$ In that case, one of the goals of cervical decompression of the spinal lipoma was to relieve any compression of the cord that may have been contributing to advancing scoliosis. Despite surgical decompression, however, the patient in their case proceeded to display worsening of the scoliosis and a separate corrective surgery for this deformity was being planned at the time of publication in 2011. Our patient underwent one corrective scoliosis surgery in 2008. Despite this intervention, his scoliosis continues to progress and the patient may require a second surgical procedure to deal with this deformity.
The natural history of our patient's disease course over twelve years appears to be consistent with the few cases that have been reported in older children. The extensive spinal lipoma has not progressed over the 12 years and in fact has shown regression intracranially. In 1999 his lipoma protruded 5 millimetres into the posterior fossa. In 2001 there was 20 millimetres of protrusion of the rostral component into the posterior fossa. The most recent images obtained in 2011 demonstrated a regression of the intracranial component of the lipoma, with only 6 millimetres of extension into the posterior fossa. The caudal extent of the tumour has been stable all along, with persistent extension to the L3 level. (Figure 4) Neurologically, our patient's neurological deficits have been quite stable over time on clinical examination. His seizure disorder that existed from birth did remit, however, and resurfaced in the past year and is currently being treated with anticonvulsants. Our patient has had unchanged normal cognitive function despite his neurological findings. The arachnoid cyst has not evolved and our patient has not had any further intracranial lesions identified in serial imaging over his 12 years.

\section{Conclusion}

While impressive on imaging, it may not be necessary to remove ECCL patients' intraspinal lipoma. In our case, it was chosen not to operate on the lipoma, and the patient has remained remarkably stable from a neurological point of view for over 12 years of follow-up. Patients with ECCL and an intraspinal lipoma appear to be at high risk of progressive scoliosis.

\section{REFERENCES}

1. Moog U. Encephalocraniocutanous lipomatosis. J Med Genetics. 2009;46:721-9.

2. Ayer R., Zourous A. Encephalocraniocutaneous lipomatosis: a review of its clinical pathology and neurosurgical indications. J Neurosurg Pediatrics. 2011;8:316-20.

3. Hunter A. Oculocerebrocutaneous and encephalocraniocutaneous lipomatosis syndromes: blind men and an elephant or separate syndromes? Am J Medical Genetics. 2006;140A:709-26.

4. Fishman M. Encephalocraniocutaneous lipomatosis. J Child Neurol. 1987;2:186.

5. Le Feuvre D, Semple P, Peter J. Intradural cervical lipomas with intracranial extension: a management strategy based on a case report and review of the literature. Br J Neurosurg. 2004;18(4): 385-8. 